\title{
Sliding-Mode Observer for Speed and Position Sensorless Control of Linear-PMSM
}

\author{
Saeed Masoumi Kazraji (Doctoral Student, University of Tabriz), \\ Ramin Bavili Soflayi (Master Student, University of Tabriz), \\ Mohammad Bagher Bannae Sharifian (Professor, University of Tabriz)
}

\begin{abstract}
The paper presents a sliding-mode observer that utilizes sigmoid function for speed and position sensorless control of permanent-magnet linear synchronous motor (PMLSM). In conventional sliding mode observer method there are the chattering phenomenon and the phase lag. Thus, in order to avoid the usage of the low pass filter and the phase compensator based on back EMF, in this paper a sliding mode observer with sigmoid function for detecting the back EMF in a PMLSM is designed to estimate the speed and the position of the rotor. Most of conventional sliding mode observers use sign or saturation functions which need low pass filter in order to detect back electromotive force (back EMF). In this paper a sigmoid function is used instead of discontinuous sign function to decrease undesirable chattering phenomenon. By reducing the chattering, detecting of the back EMF can be made directly from switching signal without any low pass filter. Thus the delay time in the proposed observer is eliminated because of the low pass filter. Furthermore, there is no need to compensate phase fault in position and speed estimating of linear-PMSM. Advantages of the proposed observer have been shown by simulation with MATLAB software.
\end{abstract}

Keywords - Permanent magnet motors; Motor drives; Observers; Sliding mode control; Sensorless control.

\section{INTRODUCTION}

Permanent magnet linear synchronous motors (PMLSMs) are used widely in systems which need linear drives. They are good alternatives for rotary motors operating with mechanical converters to produce linear movement. The main reasons of absorbing many attentions by PMLSMs are larger power density, high efficiency as well as better time response due to the magnetic field that is absent in linear induction motors [1] - [5]. Researches of vector control are divided into two major groups: 1) Field oriented control approach which is analyzed completely in [5]. This method is sensitive to variation of parameters and its equations are obtained from complex transformations. 2) Direct torque and flux control (DTC and DFC) approach which is accepted as a form of vector control methods beside vector current control. This control approach carries out a strong operation in both of transient and steady state and has a structure which responses fast and robust to the variation of parameters. It does not have complexity of first method and can be feasible [6], [7]. There are three techniques for direct torque and flux control: 1) Switching table, 2) Direct self-control, 3) Space vector modulation. From these mentioned method, switching table is widely used because it is easily feasible. Furthermore it does not need to complicate the technique of modulation. In [8], [9]
DTC approach for rotary synchronous motor has been turned into a PMLSM successfully. This control method pays special attention to estimating the flux, speed and position of the motor. There are two ways for estimating the flux: 1) Estimation based on the current of motor, 2) Estimation based on voltage of motor. The drawback of the first way is that a change of rotary speed leads to a change in the values of parameters. In the second one, the linkage flux is given directly by the electromotive force with an integrator, so there is no need in speed signal. It means that the second way is better and more complete than the first one [7], [8].

Sensorless control is divided into two main types: 1) Estimation with the use of observers, 2) Signal injection method. Comparative methods, Kalman filter and sliding mode are some of estimating methods that are based on observers. In general, the first type methods depend on the precision of the motor's model, especially the comparative methods. In other words, as the model of the motor is accurate, efficacy of this method will be improved. Kalman filter method requires a large amount of calculations. In order to reduce the time of the computations, the system must be improved by the hardware, but it is not affordable. Among the mentioned methods for estimation based on observers, sliding mode with sign function has a simple algorithm and is firm against disturbances, fault of parameters and noise. In this way, the back EMF is given by sliding mode observer. But, they also have high frequency components, though they cannot be used to compute the velocity and position of the rotor. In conventional sliding mode observers, a low pass filter is used for operation of filtering, but using of the low pass filter causes a lag of phase that depends on the angular frequency of the input signal and on the cut frequency of the filter. In order to compensate the phase lag completely, the received information from the real angular velocity must be used and the information given by the estimated angular velocity is not enough for compensating [10] - [14].

In sliding mode observer, the dynamic behavior of the system is determined by the switching level and is independent of non-deterministic events and exoteric faults. Experimentally, the constraint of the switching frequency leads to the fact that the states of the system do not stay on the switching level and fluctuate around it. These fluctuations are called chattering which is an undesirable phenomenon. It increases control activities and excites the high frequency dynamics of the system, so that the low pass filter is needed for decreasing the chattering phenomenon effects. 
As it is mentioned above, the conventional sliding mode observer method has the chattering phenomenon and the phase lag. Thus, in order to avoid the using of the low pass filter and the phase compensator based on back EMF, in this paper a sliding mode observer with sigmoid function for detecting the back EMF in a PMLSM is designed to estimate the speed and the position of the rotor. Overview of direct thrust force control \& the mathematical model of PMLSM is given in section 2. In section 3 the proposed sliding mode observer design is presented. Section 4 presents estimation rotor position and velocity using the proposed method. The simulation result is presented in section 5. The last section 6 presents summary and conclusions.

\section{DiRECT THRUST ForCE CONTROL \& MATHEMATICAL MODEL OF PMLSM}

Direct thrust force control (DTFC) method is a version of DTC for the linear motors. Its only difference compared with common DTC is that it deals with linear velocity and thrust force instead of the angular velocity and torque. The DTC/DTFC provides quick electromagnetic torque / thrust force response of the motor. The common vector control utilizes the stator current vector to alter the torque / thrust force. In contrast, the control variable of the DTC is the stator flux linkage vector. The structure of the DTFC controller is presented in Fig.1. As it is seen from this picture, the regulation of the flux linkage is finally provided by VSI by means of the optimal operation of its power switches [7], [13].

In order to obtain the PMLSM model in dq reference frame, first the stator voltage equation should be introduced [6]:

$$
\begin{gathered}
u_{d}(t)=R i_{d}+\frac{d \psi_{d}}{d t}-\omega \psi_{q}, \\
u_{q}(t)=R i_{q}+\frac{d \psi_{q}}{d t}-\omega \psi_{d} \text { and } \\
\omega=\frac{\pi}{\tau} v_{\text {lin }},
\end{gathered}
$$

where $u_{d}$ and $u_{q}$ are components of voltage space vector, but $i_{d}$ and $i_{q}$ are the components of current space vector across the $d$ and $q$ axis. At the same time $R$ is the phase resistance of armature.

The linkage fluxes $\psi_{d}$ and $\psi_{q}$ in the above equations are yielded from these ones:

$$
\begin{gathered}
\psi_{d}=L_{d} i_{d}+\psi_{P M} \text { and } \\
\psi_{q}=L_{q} i_{q},
\end{gathered}
$$

where $\mathrm{L}_{\mathrm{d}}$ and $\mathrm{L}_{\mathrm{q}}$ are the inductance of armature and $\psi_{P M}$ is the linkage flux of permanent magnet.

The input three phase instant power of armature $p$ is obtained by the following:

$$
P=u_{A} i_{A}+u_{B} i_{B}+u_{C} i_{C}=\frac{3}{2}\left(u_{d} i_{d}+u_{q} i_{q}\right),
$$

where $u_{A}, u_{B}$ and $u_{C}$ are the instant phase voltages, $i_{A}, i_{B}$ and $i_{C}$ are the instant phase currents, $u_{d}$ and $u_{q}$ are the $d$ and $q$ axis voltages, $i_{d}$ and $i_{q}$ are the $d$ and $q$ axis currents.

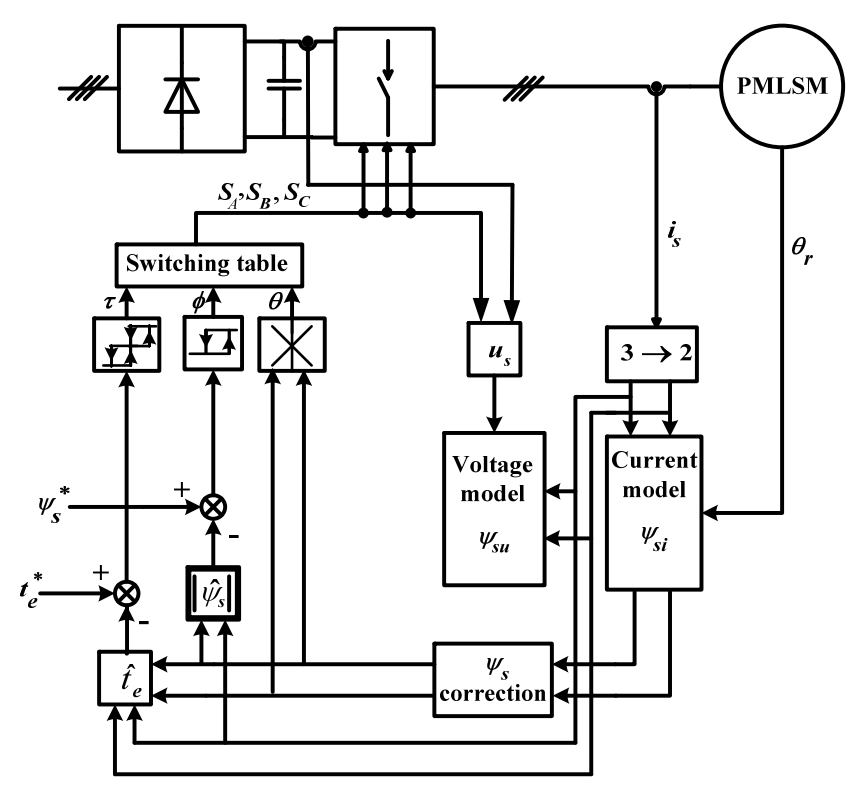

Fig. 1. DTFC block diagram.

By using the stator voltage equations, the power equation is expressed as:

$$
\begin{gathered}
u_{d} i_{d}+u_{q} i_{q}=R i_{d}^{2}+\frac{d \psi_{d}}{d t} i_{d}+R i_{q}^{2}+\frac{d \psi_{q}}{d t} i_{q}+ \\
+\omega\left(\psi_{d} i_{d}-\psi_{q} i_{q}\right)
\end{gathered}
$$

where the last component is the electromagnetic power of two poles synchronous machine in each phase. In three phase machines, the power equation is:

$$
P_{e l m}=\frac{3}{2} \omega\left(\psi_{d} i_{q}-\psi_{q} i_{d}\right)=\frac{3}{2}\left(\psi_{P M}+\left(L_{d}-L_{q}\right) i_{d}\right) i_{q} .
$$

The electromagnetic thrust of PMLSM $F_{\text {thrust }}$ with $p$ pair poles is concluded from (3) and (8) as follows:

$$
\begin{aligned}
F_{\text {thrust }}=\frac{3}{2} p \frac{\pi}{\tau}\left(\psi_{d} i_{q}-\psi_{q} i_{d}\right)= \\
=\frac{3}{2} p \frac{\pi}{\tau}\left(\psi_{P M}+\left(L_{d}-L_{q}\right) i_{d}\right) i_{q} .
\end{aligned}
$$

Equations (1)-(5) and (9) is the base of PMLSM model and its equations are as follows:

$$
\begin{aligned}
& \frac{d i_{\alpha}}{d t}=-\frac{R_{s}}{L_{s}} i_{\alpha}-\frac{1}{L_{s}} e_{\alpha}+\frac{1}{L_{s}} u_{\alpha} \\
& \frac{d i_{\beta}}{d t}=-\frac{R_{s}}{L_{s}} i_{\beta}-\frac{1}{L_{s}} e_{\beta}+\frac{1}{L_{s}} u_{\beta} \\
& e_{\alpha}=-\psi_{P M} \omega \sin \theta \\
& e_{\beta}=-\psi_{P M} \omega \cos \theta
\end{aligned}
$$


In motion control application, nonlinear elements in a linear motor cause insignificant errors in tracking or increase settling time. So, they must be considered carefully to prevent their negative effects. The dynamic behaviour of the linear motor can be expressed as:

$$
F_{\text {thrust }}=m_{\text {tot }} \frac{d v_{\text {lin }}}{d t}+F_{\text {load }}(t)+F_{\text {friction }}\left(v_{\text {lin }}\right)+F_{\text {disturb }}(x),
$$

where $m_{\text {tot }}$ is the total mass of the mover and the load, $v_{\text {lin }}$ is the linear velocity of the mover, $F_{\text {friction }}$ is the friction force which is caused by viscosity, coulomb and static effects, $F_{\text {load }}$ is the extra force which is produced by the load and $F_{\text {disturb }}$ is the force which includes cogging and end effect.

\section{The Proposed SLIDING Mode ObServer}

The equations which are used in a conventional sliding mode observer are written as (10). By using mathematical model of PMLSM and defining slip level as $S=i_{s^{-}} i_{s}=0$ the equations are written as follows [15]-[17]

$$
\begin{aligned}
& L_{s} \frac{d i_{\alpha}}{d t}=-R_{s} \hat{i_{\alpha}}+u_{\alpha}-k \operatorname{sign}\left(\hat{i_{\alpha}}-i_{\alpha}\right) \\
& L_{s} \frac{d i_{\beta}}{d t}=-R_{s} \hat{i_{\beta}}+u_{\beta}-k \operatorname{sign}\left(\hat{i_{\beta}}-i_{\beta}\right) .
\end{aligned}
$$

In the above analysis due to the chattering reduction it is possible to substitute a continuous function for the sign function. The continuous function is defined as:

$$
F(x)=\left[\frac{2}{\left(1+e^{-\alpha x}\right)}\right]-1,
$$

where $\alpha$ is the adjustable value. By defining the continuous function as above, the sliding mode observer equation is rewritten as:

$$
\begin{aligned}
& L_{s} \frac{d \hat{i_{\alpha}}}{d t}=-R_{s} \hat{i_{\alpha}}+u_{\alpha}-k F\left(\hat{i_{\alpha}}-i_{\alpha}\right) \\
& L_{s} \frac{d \hat{i_{\beta}}}{d t}=-R_{s} \hat{i_{\beta}}+u_{\beta}-k F\left(\hat{i_{\beta}}-i_{\beta}\right) .
\end{aligned}
$$

For stability analysis of the above sliding mode observer, Lyapunov function is chosen as:

$$
V=\frac{1}{2} S(X)^{T} S(X)
$$

Requisite condition for stability of sliding mode observer is obtained as follows:

$$
\dot{V}=S(X)^{T} \dot{S}(X) \leq 0
$$

By subtracting (10) from (14), the error equation is concluded:

$$
\begin{aligned}
& L_{s}\left[\frac{d S_{\alpha}(X)}{d t}\right]=-R_{s} S_{\alpha}(X)+e_{\alpha}-k F\left(\hat{i_{\alpha}}-i_{\alpha}\right) \\
& L_{s}\left[\frac{d S_{\beta}(X)}{d t}\right]=-R_{s} S_{\beta}(X)+e_{\beta}-k F\left(\hat{i}_{\beta}-i_{\beta}\right) .
\end{aligned}
$$

$S(X)$ is defined as:

$$
S(X)=\left[\begin{array}{l}
S_{\alpha}(X) \\
S_{\beta}(X)
\end{array}\right]=\left[\begin{array}{c}
\hat{i_{\alpha}}-i_{\alpha} \\
\hat{i_{\beta}}-i_{\beta}
\end{array}\right] .
$$

By derivative from the above equation, the stability condition is as follows:

$$
\begin{aligned}
& \dot{V}=S(X)^{T} \dot{S}(X)=S_{\alpha} \dot{S}_{\alpha}+S_{\beta} \dot{S}_{\beta} \\
& =\frac{1}{L_{s}}\left[\left(\hat{i_{\alpha}}-i_{\alpha}\right) e_{\alpha}-k\left(\hat{i_{\alpha}}-i_{\alpha}\right) F\left(\hat{i_{\alpha}}-i_{\alpha}\right)\right] \\
& +\frac{1}{L_{s}}\left[\left(\hat{i_{\beta}}-i_{\beta}\right) e_{\beta}-k\left(\hat{i_{\beta}}-i_{\beta}\right) F\left(\hat{i_{\beta}}-i_{\beta}\right)\right] \\
& -\frac{R s}{L_{s}}\left[\left(\hat{i_{\alpha}}-i_{\alpha}\right)^{2}+\left(\hat{i_{\beta}}-i_{\beta}\right)^{2}\right] \leq 0
\end{aligned}
$$

Thereupon:

$$
k>\max \left(\left|e_{\alpha}\right|\left|e_{\beta}\right|\right) \text {. }
$$

As $k$ is high enough, the asymptotic stability and slip movement seems certain. When the system is getting to the slip level:

$$
\dot{S}(X)=S(X)=0 .
$$

By putting above equation in (17), which is based on equivalent control way, the following are given:

$$
\begin{aligned}
& e_{\alpha}=k F\left(\hat{i}_{\alpha}-i_{\alpha}\right) \\
& e_{\beta}=k F\left(\hat{i}_{\beta}-i_{\beta}\right) .
\end{aligned}
$$

\section{Estimation Rotor Position AND SPEED With PROPOSED METHOD}

Back EMF can be obtained by the described sliding mode observer, but the signals still include high frequency components. Therefore, they can not be used directly to estimate the rotor position and speed. In a conventional sliding mode observer a low pass filter is used for filtering operation causing the phase lag that depends on the cut frequency and the input signal angular one. In order to compensate the phase lag completely the acquired information from the real linear speed must be used and the information given by the estimated linear speed is not enough for the compensating. Thus, to avoid the use of the low pass filter and the phase compensator based on back EMF, a sliding mode observer for detecting the back EMF in a PMLSM is designed to estimate the speed and the position of the rotor. As the changes of the motor linear speed are less than those of the stator's current, it is assumed that $\omega=0$. So, back EMF of PMLSM can be expressed as:

$$
\begin{aligned}
& \frac{d e_{\alpha}}{d t}=-\omega e_{\beta} \\
& \frac{d e_{\beta}}{d t}=-\omega e_{\alpha} .
\end{aligned}
$$




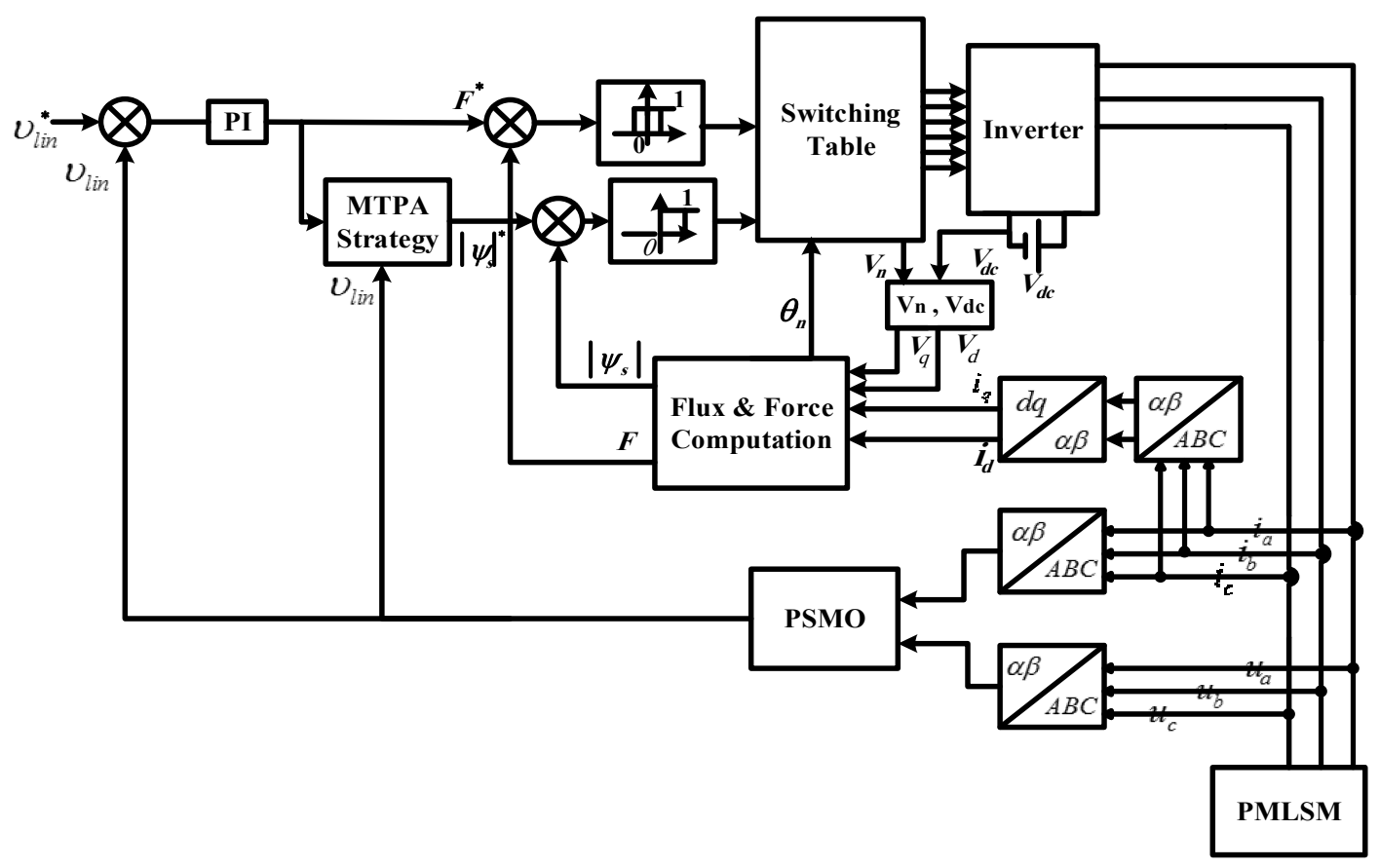

Fig. 2. Block diagram of the PMLSM sensorless control.

In consideration of above equation, the back EMF observer is designed which is based on the following:

$$
\begin{aligned}
& \frac{d \hat{e}_{\alpha}}{d t}=-\hat{\omega} \hat{e}_{\beta}-l\left(\hat{e}_{\alpha}-e_{\alpha}\right) \\
& \frac{d \hat{e}_{\beta}}{d t}=-\hat{\omega} \hat{e}_{\beta}-l\left(\hat{e}_{\beta}-e_{\beta}\right) \\
& \frac{d \hat{\omega}}{d t}=\left(\hat{e}_{\alpha}-e_{\alpha}\right) \hat{e}_{\beta}-\left(\hat{e}_{\beta}-e_{\beta}\right) \hat{e}_{\alpha},
\end{aligned}
$$

where $l$ is the gain of the observer $(>1)$.

The equation of the observer error can be obtained by subtracting (23) from (24):

$$
\begin{aligned}
\frac{d \tilde{e}_{\alpha}}{d t} & =-\tilde{\omega} \hat{e}_{\beta}-\omega \tilde{e}_{\beta}-l \tilde{e}_{\alpha} \\
\tilde{\omega}=\omega-\omega \frac{d \tilde{e}_{\beta}}{d t} & =-\tilde{\omega} \hat{e}_{\alpha}-\omega \tilde{e}_{\alpha}-l \tilde{e}_{\beta} \\
\frac{d \tilde{\omega}}{d t} & =\tilde{e}_{\alpha} \hat{e}_{\beta}-\tilde{e}_{\beta} \hat{e}_{\alpha},
\end{aligned}
$$

where $\tilde{e}_{\alpha}=\hat{e}_{\alpha}-e_{\alpha}, \tilde{e}_{\beta}=\hat{e}_{\beta}-e_{\beta}$ and $\widetilde{\omega}=\omega-\omega$.

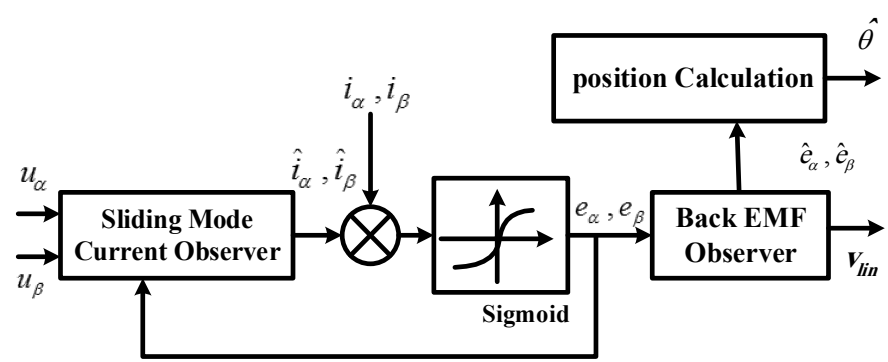

Fig. 3. Block diagram of the purposed sliding mode observer (PSMO).
Since (24) is stable, it is possible to define Lyapunov function as follows:

$$
V=\frac{\left(\tilde{e}_{\alpha}^{2}+\tilde{e}_{\beta}^{2}+\tilde{\omega}^{2}\right)}{2} .
$$

Derivative from the above equation is led to:

$$
\dot{V}=\tilde{e}_{\alpha} \dot{\tilde{e}}_{\alpha}+\tilde{e}_{\beta} \dot{\tilde{e}}_{\beta}+\tilde{\omega} \dot{\tilde{\omega}}
$$

Inserting (25) in (27) produces:

$$
\dot{V}=-l\left(\tilde{e}_{\alpha}^{2}+\tilde{e}_{\beta}^{2}\right) \leq 0 \text {. }
$$

The mentioned equation proves the fact that the proposed back EMF observer is asymptotic stable. Therefore, the use of the back EMF obtained from the observer, as well as the use of the relationship of the back EMF and state of the rotor, allows estimating the position signal as:

$$
\hat{\theta}=-\arctan \left(\frac{\hat{e}_{\alpha}}{\hat{e}_{\beta}}\right) .
$$

The speed is obtained easily by using an integrator in the observer. The overall scheme of the PMLSM sensorless control and the block diagram of the purposed sliding mode observer (PMSO) are shown in sequence in Fig. 2 and Fig. 3. In the first one, it must be noticed that the input of the sliding mode observer comes from the motor's voltage and the output voltages of the current loop $\mathrm{u}_{\alpha}{ }^{*}$ and $\mathrm{u}_{\beta}{ }^{*}$ are not used. Usage of 
$\mathrm{u}_{\alpha}$ and $\mathrm{u}_{\beta}$ reduces relatively the dead time effect in the inverter. So, the motor's voltage is obtained more precisely and thus estimates accuracy of the motor position and speed are improved.

\section{V.SIMULATION RESULTS}

The parameters given by the manufacturer of the motor [18] and inverter parameters are presented in Table I.

The step function is considered as a reference signal. The speed which is estimated by a sliding mode observer has been shown in Fig. 4. Chattering phenomenon in these types of observers which use discontinuous sign function is seen obviously [19], [20].

The speed which is estimated by the proposed sliding mode observer has been shown in Fig 5. As it is seen, comparison with conventional observers, chattering phenomenon is reduced effectively. Moreover, low pass filter and phase lag compensator are not required.

Fig.6 shows acceleration of the mover in order for a conventional observer and the proposed sliding mode. Fig. 7 shows the force which has been developed by the mover considering the end effects of the cogging and friction forces. Comparison of Fig. 7 (a) [16] with Fig. 7 (b) shows that the reference and estimated forces in system which uses the proposed sliding mode observer is more accurate than others which use conventional ones, because choosing continuous function instead of discontinuous sign function leads to eliminate chattering phenomenon.

Fig. 8 shows the current of the system which uses the proposed sliding mode observer during accelerating time of the mover at the stability speed and when acceleration of the mover is reduced.

TABLE I

PARAMETERS OF THE MOTOR MODEL AND INVERTER

\begin{tabular}{|l|l|l|}
\hline Symbol & Value & Parameter \\
\hline $\mathrm{R}$ & $1.6 \Omega$ & Phase resistance \\
\hline $\mathrm{L}_{\mathrm{d}}$ & $0.013 \mathrm{H}$ & d-axis inductance \\
\hline $\mathrm{L}_{\mathrm{q}}$ & $0.013 \mathrm{H}$ & q-axis inductance \\
\hline$p$ & 1 & Number of pole pairs \\
\hline$\tau$ & $0.012 \mathrm{~m}$ & PM pole pitch \\
\hline$\psi_{P M}$ & $0.237 \mathrm{~Wb}$ & PM flux linkage \\
\hline $\mathrm{U}_{\mathrm{N}}$ & $560 \mathrm{~V}$ & Rated voltage inverter \\
\hline $\mathrm{P}_{\mathrm{N}}$ & $3.5 \mathrm{~kW}$ & Rated power \\
\hline $\mathrm{F}_{\mathrm{N}}$ & $1800 \mathrm{~N}$ & Rated force \\
\hline $\mathrm{V}_{\max }$ & $4.5 \mathrm{~m} / \mathrm{s}$ & Peak speed \\
\hline
\end{tabular}

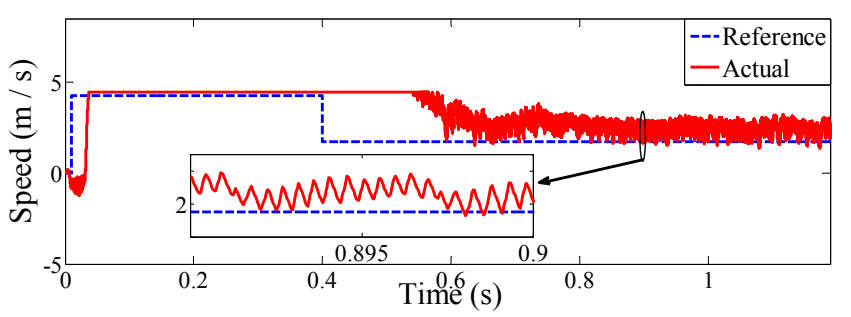

Fig. 4. Speed reference signal and actual speed of the motor by sliding mode observer.

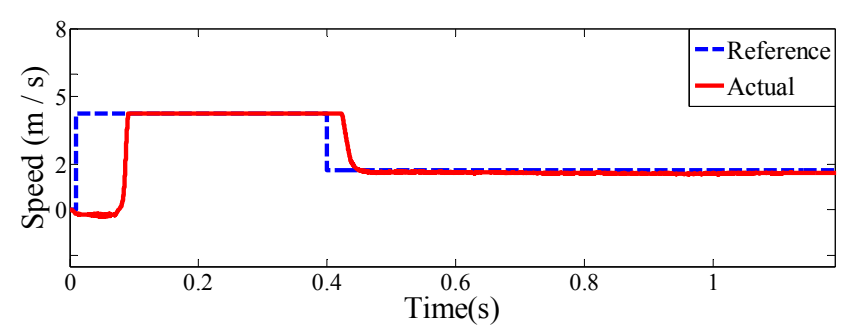

Fig. 5. Speed reference signal and speed actual of the motor by the proposed sliding mode observer.

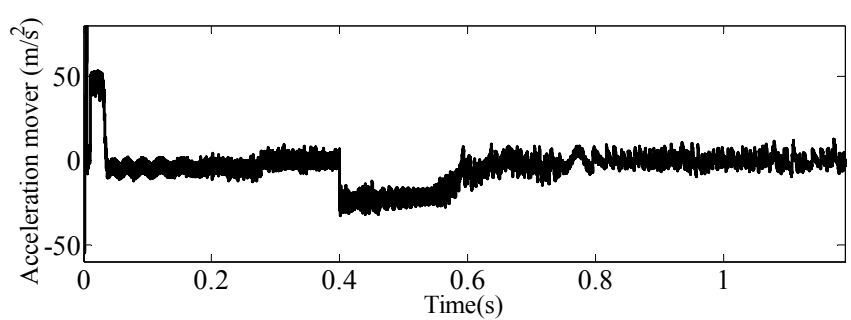

(a)

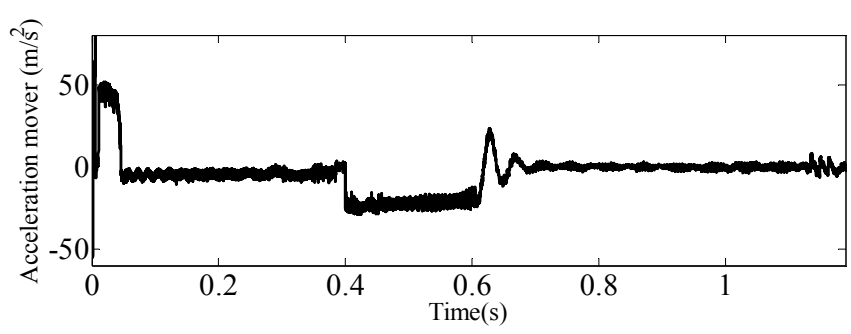

(b)

Fig. 6. Acceleration of the mover (a) by using a conventional sliding mode observer (b) by using the proposed sliding mode observer. 


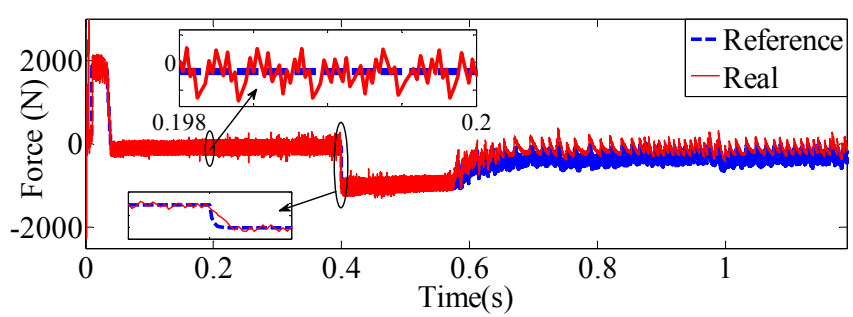

(a)

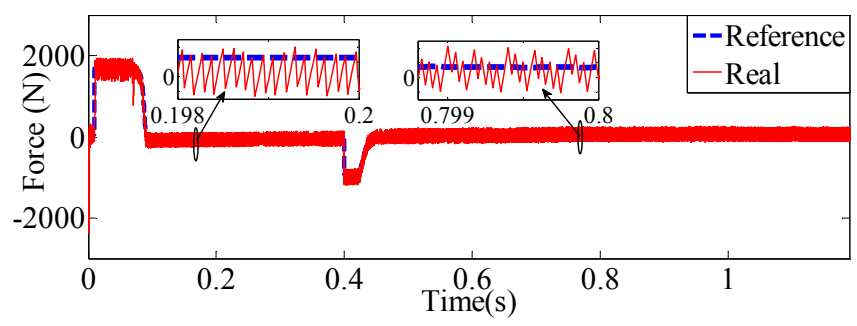

(b)

Fig. 7. The produced force of the mover (a) by using a conventional sliding mode observer (b) by using the proposed sliding mode observer.

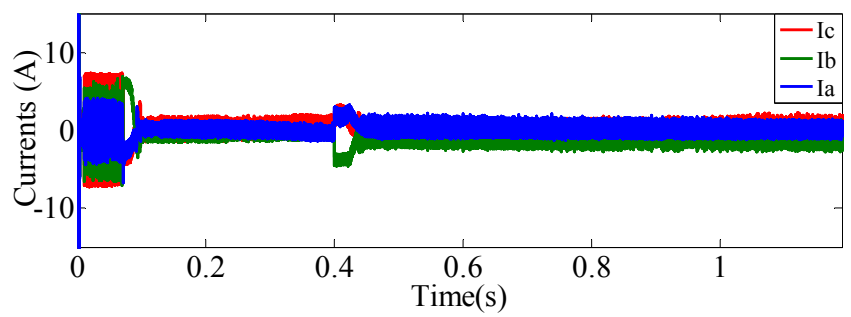

Fig. 8. The currents of PMLSM which uses the proposed sliding mode observer.

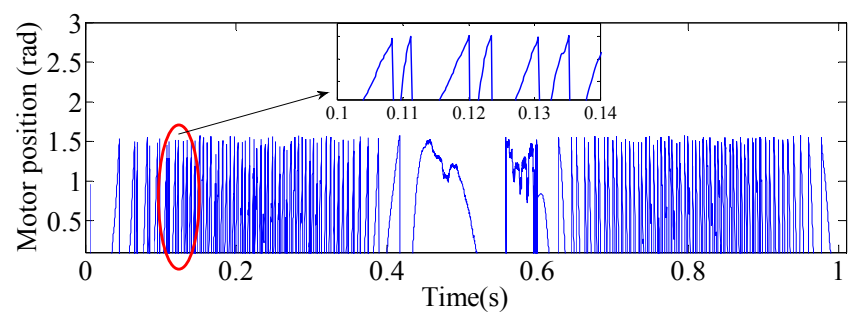

Fig. 9. Position response for the motor.

The position which is estimated by the proposed sliding mode observer has been shown in Fig. 9. It is possible to see that the phenomenon of chattering of the estimated rotor position is reduced. Moreover, low pass filter and phase lag compensator are not required.

\section{CONCLUSIONS}

In this paper the improved sliding mode observer which uses sigmoid function was proposed for PMLSM sensorless control. The paper illustrates that the use of sigmoid function instead of sign function, that is usual in sliding mode observers, leads to reduce undesirable chattering phenomenon effectively. Reducing chattering phenomenon results in the fact that back EMF can be detected directly from switching signal without any need to low pass filter. So the delay time caused by the presence of low pass filter in the proposed observer is omitted. Moreover, there is no need to compensate phase fault in estimated position. Advantages of the proposed observer over conventional ones have been shown with the results obtained from simulation in MATLAB/Simulink software.

\section{REFERENCES}

[1] J. Batelaan, "A Linear Motor Design Provides Close and Secure Vehicle Separation of Many Transit Vehicles on a Guideway," IEEE Transactions on Industrial Electronics, vol. 54, no. 3, pp. 1778-1782, Jun. 2007.

[2] L. N. Tutelea, M. C. Kim, M. Topor, J. Lee, and I. Boldea, "Linear Permanent Magnet Oscillatory Machine: Comprehensive Modeling for Transients With Validation by Experiments," IEEE Transactions on Industrial Electronics, vol. 55, no. 2, pp. 492-500, 2008.

[3] M. Boussak, "Implementation and Experimental Investigation of Sensorless Speed Control With Initial Rotor Position Estimation for Interior Permanent Magnet Synchronous Motor Drive," IEEE Transactions on Power Electronics, vol. 20, no. 6, pp. 1413-1422, Nov. 2005.

[4] Y.-S. Kung, "Design and Implementation of a High-Performance PMLSM Drives Using DSP Chip," IEEE Transactions on Industrial Electronics, vol. 55, no. 3, pp. 1341-1351, Mar. 2008.

[5] T.-H. Liu, Y.-C. Lee, and Y.-H. Chang, "Adaptive controller design for a linear motor control system," IEEE Transactions on Aerospace and Electronic Systems, vol. 40, no. 2, pp. 601-616, Apr. 2004.

[6] Y.-W. Zhu and Y.-H. Cho, "Thrust Ripples Suppression of Permanent Magnet Linear Synchronous Motor," IEEE Transactions on Magnetics, vol. 43, no. 6, pp. 2537-2539, Jun. 2007.

[7] I. Takahashi and Y. Ide, "Decoupling control of thrust and attractive force of a LIM using a space vector control inverter," IEEE Transactions on Industry Applications, vol. 29, no. 1, pp. 161-167, 1993.

[8] X. del Toro Garcia, A. Arias, M. G. Jayne, and P. A. Witting, "Direct Torque Control of Induction Motors Utilizing Three-Level Voltage Source Inverters," IEEE Transactions on Industrial Electronics, vol. 55, no. 2, pp. 956-958, 2008.

[9] F. Morel, J.-M. Retif, X. Lin-Shi, and C. Valentin, "Permanent Magnet Synchronous Machine Hybrid Torque Control," IEEE Transactions on Industrial Electronics, vol. 55, no. 2, pp. 501-511, 2008.

[10] K. C. Veluvolu and Y. C. Soh, "High-Gain Observers With Sliding Mode for State and Unknown Input Estimations," IEEE Transactions on Industrial Electronics, vol. 56, no. 9, pp. 3386-3393, Sep. 2009.

[11] Q. D. Nguyen and S. Ueno, "Improvement of sensorless speed control for nonsalient type axial gap self-bearing motor using sliding mode observer," in 2010 IEEE International Conference on Industrial Technology, 2010, pp. 373-378.

[12] A. Jaafar, E. Godoy, P. Lefranc, X. L. Shi, A. Fayaz, and N. Li, "Nonlinear sliding mode observer and control of high order DC-DC converters," in IECON 2010 - 36th Annual Conference on IEEE Industrial Electronics Society, 2010, pp. 180-186.

[13] M. Abroshan, K. Malekian, J. Milimonfared, and B. Abdi Varmiab, “An optimal direct thrust force control for interior Permanent Magnet Linear Synchronous Motors incorporating field weakening," in 2008 International Symposium on Power Electronics, Electrical Drives, Automation and Motion, 2008, pp. 130-135. 
[14] A. Hijazi, X. Lin Shi, A. Zgorski, and L. Sidhom, "Adaptive sliding mode observer-differentiator for position and speed estimation of Permanet Magnet Synchronous Motor," in 3rd IEEE International Symposium on Sensorless Control for Electrical Drives (SLED 2012), 2012, pp. 1-5.

[15] Z. Qiao, T. Shi, Y. Wang, Y. Yan, C. Xia, and X. He, "New SlidingMode Observer for Position Sensorless Control of Permanent-Magnet Synchronous Motor," IEEE Transactions on Industrial Electronics, vol. 60, no. 2, pp. 710-719, Feb. 2013

[16] Y. Zhao, W. Qiao, and L. Wu, "An Adaptive Quasi-Sliding-Mode Rotor Position Observer-Based Sensorless Control for Interior Permanent Magnet Synchronous Machines," IEEE Transactions on Power Electronics, vol. 28, no. 12, pp. 5618-5629, Dec. 2013.

[17] S. Chi, Z. Zhang, and L. Xu, "Sliding-Mode Sensorless Control of Direct-Drive PM Synchronous Motors for Washing Machine Applications," IEEE Transactions on Industry Applications, vol. 45, no. 2, pp. 582-590, 2009.

[18] TECNOTION B.V., "A primer of Tecnotion Linear Motors," 2008. [Online]. Available: http://www.tecnotion.com/files/Manuals/Basic Information_LM_EN_V21_20080912.pdf.

[19] J. Chu, Y. Hu, W. Huang, M. Wang, J. Yang, and Y. Shi, "An improved sliding mode observer for position sensorless vector control drive of PMSM," in 2009 IEEE 6th International Power Electronics and Motion Control Conference, 2009, pp. 1898-1902.

[20] H. Kim, J. Son, and J. Lee, "A High-Speed Sliding-Mode Observer for the Sensorless Speed Control of a PMSM," IEEE Transactions on Industrial Electronics, vol. 58, no. 9, pp. 4069-4077, Sep. 2011.

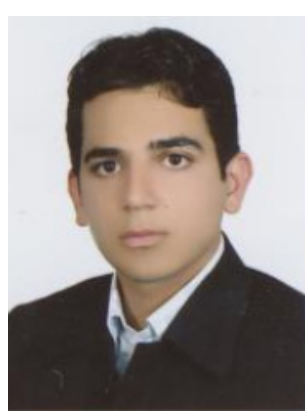

Saeed Masoumi Kazraji was born in Tehran, Iran in 1989. He received the B.Sc. degree from the Sbzevar University of Tarbiat Moallem, Mashhad, Iran in 2011 and the M.Sc. degree from University of Tabriz, Tabriz, Iran in 2013 both in Electrical Power Engineering. He is currently pursuing the Ph.D. University of Tabriz. He has a degree in electrical engineering (machine and electric drives). His research interests include drive and motion control of electric machines, linear and rotary machine design, renewable energy in electric vehicle drive application, and power electronic converters.

Address: University of Tabriz, Faculty of Electrical and Computer Engineering, Iran, Tabriz, 29 Bahman blvd.

E-mail: masoumikazraji@tabrizu.ac.ir

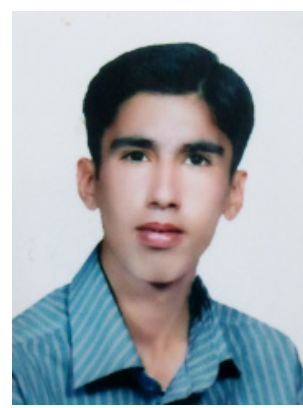

Ramin Bavili was born in Tabriz, Iran 1990. $\mathrm{He}$ received the B.Sc. degree in Electrical Engineering from Islamic Azad University, Ilkhichi Branch, in 2012. He is currently pursuing his M.Sc. degree in Electrical Engineering at University of Tabriz. His current research of electric drive and interests include power systems and intelligent search methods.

Address: University of Tabriz, Faculty of Electrical and Computer Engineering, Iran, Tabriz, 29 Bahman blvd.

E-mail: ramin.bavil90@gmail.com

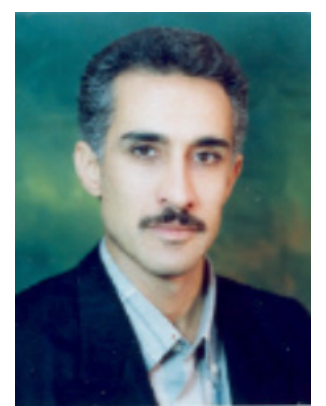

Mohammad Bagher Bannae Sharifian (1965) studied Electrical Power Engineering at the University of Tabriz, Tabriz, Iran. He received the B.Sc. and M.Sc. degrees in 1989 and 1992 respectively from University of Tabriz. In 1992 he joined the Electrical Engineering Department of the University of Tabriz as a lecturer. He received Ph.D. degree in Electrical Engineering from the same University in 2000. In 2000 he rejoined the Electrical Power Department of Faculty of Electrical and Computer Engineering of the same university as Assistant Professor. He is currently Professor of the mentioned Department. His research interests are in the areas of design, modeling and analysis of electrical machines, transformers, liner electric motors, and electric and hybrid electric vehicle drives.

Address: University of Tabriz, Faculty of Electrical and Computer Engineering, Iran, Tabriz, 29 Bahman blvd.

E-mail: sharifian@tabrizu.ac.ir 